CERN-TH/97-172

hep-ph/9707423

\title{
New physics in a nutshell, or Q-ball as a power plant
}

\author{
Gia Dvali卂, Alexander Kusenko and Mikhail Shaposhnikov' \\ Theory Division, CERN, CH-1211 Geneva 23, Switzerland
}

\begin{abstract}
Future experiments may discover new scalar particles with global charges and couplings that allow for solitonic states. If the effective potential has flat directions, the scalar VEV inside a large Q-ball can exceed the particle mass by many orders of magnitude. Models with low-energy supersymmetry breaking generically have both the scalars carrying some global charges, and the flat directions. The Q-ball interior can, therefore, provide an environment for exploring physics far beyond the $\mathrm{TeV}$ scale without the need for building colliders of ever-increasing energies. Some Standard Model processes, otherwise strongly suppressed, can also be studied inside the soliton, where the $\mathrm{SU}(2)$ symmetry can be restored, and the quark confinement may be absent. Baryon number violating processes catalyzed by the large VEV inside the Q-ball can provide an inexhaustible energy resource.
\end{abstract}

CERN-TH/97-172

July, 1997

\footnotetext{
* email address: dvali@mail.cern.ch

** email address: kusenko@mail.cern.ch

$\dagger$ email address: mshaposh@nxth04.cern.ch
} 


\section{Introduction}

The development of particle colliders has greatly advanced one's understanding of the fundamental laws of nature. However, it is becoming increasingly clear that growing costs and complexity of the accelerating technology will eventually stymie further exploration, unless some new paradigm will replace the concept of a collider experiment.

In this Letter we point out that the new frontiers that will be unveiled by the next generation of particle colliders, in particular the LHC, may allow for a conceptually new approach to high-energy experimentation. A remarkable byproduct of such a development can be a new, practically unlimited, source of energy.

New physics is essential for the applications we discuss. First, we assume that the new scalar particles, whose existence is anticipated in most theories beyond the Standard Model, will be found in future experiments. Second, it is necessary that the low-energy effective potential be invariant under a global U(1) symmetry. Although the baryon and lepton number conservation may suffice in principle, it is preferable for technical reasons (although not necessary) that there be a new U(1) symmetry, with respect to which all the light fermions have zero charge. Finally, the success of our idea relies on the appearance of "flat directions" in the scalar potential, as is typically the case in theories with low-energy supersymmetry. We will assume hereafter that the new physics at the electroweak scale meets all three conditions. We emphasize that supersymmetry per se is not essential for our idea, which is quite general, but the presence of scalar fields and flat directions makes supersymmetric models a natural setting for applications discussed below.

Our basic idea is that the manifestations of the high-scale physics can be attained in the interior of a relatively long-lived artificially created non-topological soliton of the Q-ball type [1, 2]. A specific property of such solitons is that the scalar VEV $\langle\phi\rangle$ inside a large Q-ball tends to take the value which minimizes the ratio of the interior energy density to $\left\langle\phi^{2}\right\rangle$, that is $U(\phi) / \phi^{2}=\min$ [2]. If the potential $U(\phi)$ has flat directions, as expected in theories with low-energy supersymmetry, then the scalar VEV inside a sufficiently large Q-ball can exceed the energy density by many orders of magnitude. Various couplings, suppressed by the powers of $\langle\phi\rangle / M_{\text {new physics }} \ll 1$ in the low-energy effective Lagrangian, become large inside the Q-ball and can mediate exotic processes, whose detection can yield information 


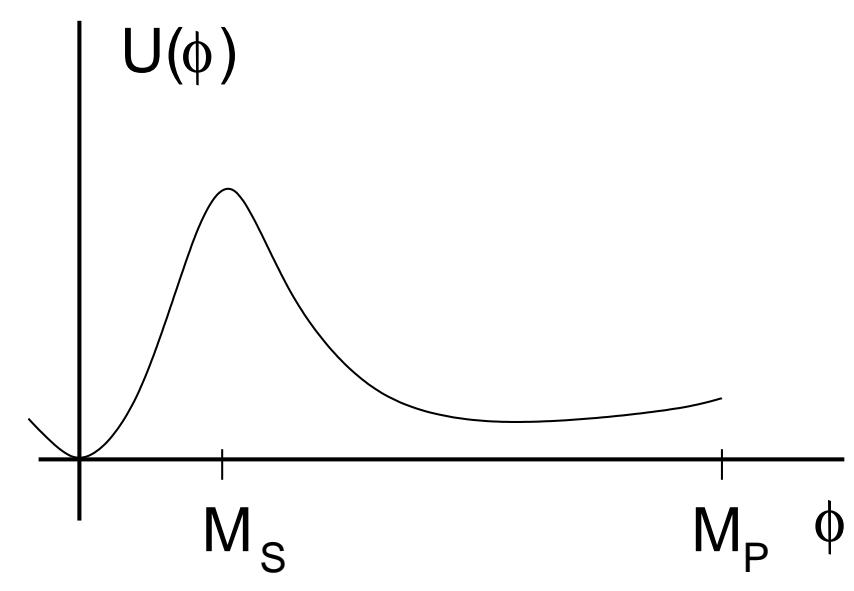

Figure 1: A potential with a flat direction lifted only by logarithmic corrections at large VEV.

about the new physics.

In addition, the ground state of matter inside a large Q-ball is different from the standard vacuum. Various processes, allowed by the Standard Model but suppressed in the vacuum with a broken electroweak symmetry, can take place in the soliton interior where the $\mathrm{SU}(2)$ can be restored, and the quark and gluons may not be confined.

A particular example of an exotic process predicted by Grand Unified and other theories, is the explicit baryon number violation. A Q-ball with a baryon number violating interior can be used to generate energy through a decay of the incoming nucleons into leptons and photons.

\section{Far-reaching Q-balls}

In this section we study non-topological solitons [1, 2] in the potential $U(\varphi)$ that is essentially flat for large values of $\varphi$, by which we mean that it grows slower than $\varphi^{2}$ (Figure 1). As discussed in the next section, such potentials are common in theories with softly-broken low-energy supersymmetry, where numerous flat directions are lifted only by the soft supersymmetry breaking terms and Planck scale suppressed operators. Although supersymmetry is not essential for what follows, it provides a natural motivation for an otherwise ad hoc potential we employ.

If $U(\varphi)$ is invariant under a global $\mathrm{U}(1)$ symmetry, $\varphi \rightarrow \exp (i \theta) \varphi$, then the theory 
admits non-topological solitons, Q-balls, which are the minima of energy in the sector of fixed charge. Such solitons can be described as time-dependent spherically symmetric lumps $\varphi(x, t)=e^{i \omega t} \varphi(r), r^{2}=\vec{x}^{2}$, where the function $\varphi(r)$ is such that it extremizes the functional 近

$$
\mathcal{E}_{\omega}=\int d^{3} x\left[\frac{1}{2}|\nabla \varphi(x)|^{2}+\hat{U}_{\omega}(\varphi(x))\right]+\omega Q .
$$

Here $Q=\frac{1}{2 i} \int \varphi^{*} \stackrel{\leftrightarrow}{\partial}_{t} \varphi d^{3} x$ is the soliton charge and $\hat{U}_{\omega}(\varphi)=U(\varphi)-\left(\omega^{2} / 2\right) \varphi^{2}$. The equation of motion for $\varphi(r)$ is that of a bounce associated with tunneling in three Euclidean dimensions in the potential $\hat{U}_{\omega}(\varphi)$ :

$$
\left\{\begin{array}{l}
\varphi^{\prime \prime}(r)+(2 / r) \varphi^{\prime}(r)=\partial \hat{U}_{\omega} / \partial \varphi \\
\varphi(0)=\varphi^{\prime}(\infty)=0 .
\end{array}\right.
$$

In the thin-wall approximation (whose applicability will be discussed below), Q-ball is approximated by an ansatz

$$
\varphi(r)= \begin{cases}\varphi_{0}, & r<R \\ 0, & r \geq R\end{cases}
$$

Both $\omega$ and $\varphi_{0}$ are found by minimizing the energy of the soliton. The value of the scalar field in the Q-ball interior is such that

$$
\frac{U(\varphi)}{\varphi^{2}}=\min \quad \text { for } \quad \varphi=\varphi_{0}
$$

Of course, for a potential of the kind shown in Figure 1, which grows slower than $\varphi^{2}$ for large $\varphi$, the thin-wall Q-ball would appear to have an infinite VEV. In fact, condition (四) determines the value of $\varphi_{0}$ only in the thin-wall limit, that is, when the gradient energy is small in comparison to the volume energy, and the ansatz (3) is appropriate. As $\varphi(0)$ increases, the thin-wall approximation breaks down for a Q-ball of a fixed charge. For a flat potential, the equation of motion that determines the shape of the soliton can be solved analytically near the origin $r=0$, where the right-hand side of the differential equation (2) is simply $\omega^{2} \varphi$. For large $r, \varphi(r) \sim \exp \left\{-m_{\varphi} r\right\}$, where $m$ is the mass of $\varphi$ near the origin. The solution can be approximated by 


$$
\varphi(r)= \begin{cases}\varphi_{0}(\sin \omega r) / \omega r, & r<R \\ \varphi_{1} \exp \left\{-m_{\varphi} r\right\}, & r \geq R,\end{cases}
$$

where the values of $\varphi_{0}, \varphi_{1}, \omega$ and $R$ are such that they minimize $\mathcal{E}_{\omega}$ in equation (1), while $\varphi(r)$ is continuous at $r=R$.

Clearly, the VEV of $\varphi$ slides along the flat direction, in accord with (匹), until the solution is way outside the thin-wall regime. In this thick-wall limit, one can rewrite $\mathcal{E}_{\omega}$ in equation (回) in terms of dimensionless variables $\xi=\omega x$ and $\psi=\varphi / \omega$ neglecting all terms in $\hat{U}_{\omega}$ except the constant term and the $-\left(\omega^{2} / 2\right) \varphi^{2}$ term ( $c f$. Refs. [4, 5]). The resulting expression for $\mathcal{E}_{\omega}$ is $\mathcal{E}_{\omega} \approx a \omega+b / \omega^{3}+\omega Q$, where $a$ and $b$ are constants independent of $\omega$. The value of $\omega$ that minimizes $\mathcal{E}_{\omega}$ depends on the charge as $\omega \propto Q^{-1 / 4}$. The size of the soliton (5) is $R \sim 1 / \omega$. Therefore, the value of the field in the Q-ball interior

$$
\varphi_{0} \propto Q^{1 / 4}
$$

We conclude that the scalar VEV inside a Q-ball can exceed the scales $M_{S}$ and $m_{\varphi}$ by many orders of magnitude, provided that the charge of a soliton is large. If the $\varphi$ particles can be produced in a collider, then, conceivably, one can build a large Q-ball by accumulating the $\mathrm{U}(1)$ charge. As the VEV of $\varphi$ slides along a flat direction, the interior of the soliton can provide access to new physics at the scales $\varphi_{0} \sim Q^{1 / 4} m_{\varphi} \gg m_{\varphi}$ for large $Q$. Of course, it would require an enormous charge to reach, for example, the GUT scale. The requisite Q-ball would have to carry charge as large as $10^{56}$ and its mass would be of order $10^{20} \mathrm{~g}$. However, conceivably, one can hope to attain the intermediate scales $\sim 10^{8} \mathrm{GeV}$ using the solitons with charges of order $N_{A} \sim 10^{23}$.

Another important property of a large Q-ball in a flat potential is that its energy grows as $E \propto Q^{3 / 4}$, rather than $Q$, which means that storing charge in the maximal size solitons is energetically preferable. This is because the Q-ball in a flat potential never becomes a thin-wall object. If the scalar VEV remained constant, the soliton mass would grow as the first power of charge. However, in our case, the VEV of $\varphi$ slides out along the flat direction as the charge increases, thus accommodating more charge at the lesser energy expense than it would be in the thin-wall limit. Hence, $E$ grows slower than $Q$. 


\section{Flat potentials in theories with broken supersymme- try}

The effective potentials of the type specified in the previous section can be naturally realized (and are, in fact, generic) in theories with supersymmetry-breaking soft masses originating at some low energy scale $\ll M_{P}$, for example, in models with gauge-mediated supersymmetry breaking [3]. To avoid the problematic supertrace relation, it is commonly assumed that the supersymmetry breaking takes place in some hidden sector, that is, the sector that has no direct couplings to the quark and lepton superfields in the superpotential. The role of this sector is to provide a superfield(s) $X$ (usually a singlets under the standard model group) with a nonvanishing scalar and auxiliary $\left(F_{X}\right)$ components. This breaks supersymmetry, and also ensures that no unbroken $R$-symmetry survives. The transmission of the supersymmetry breaking to the observable sector is due to some messenger interaction with a typical scale $M_{S}$. Supergravity, or some heavy particles charged under the standard model gauge group, can be the messengers in the so called gravity-mediated or gauge-mediated scenarios, respectively. Integrating out the messenger sector below the scale $M_{S}$, one is left with the higher dimensional couplings (suppressed by powers of $M_{S}^{-1}$ ) between the observable and the hidden sector superfields. The resulting scale of supersymmetry breaking in the observable sector is set by the ratio $F_{X} / M_{S}$. In this scenario the soft masses are "hard" below the scale $M_{S}$ and disappear above that scale. Thus, in the gravity-mediated scenarios these soft masses stay intact, up to the renormalization effects, all the way up to the Planckian energies (or the field strengths), whereas in gauge-mediated scenarios with low $M_{S}$ they die-off above the scale $M_{S}<<M_{P}$. For this reason, the behavior of the scalar potentials differs dramatically in these two cases. In our analysis we will concentrate on the effective potentials of the flat direction fields, which are very generic in supersymmetric theories.

It is well known that the MSSM and its extensions admit a variety of non-compact flat vacuum directions in the unbroken supersymmetry limit. There are certain combinations of the scalar fields (squarks and sleptons), such that $D$ and $F$ terms in the scalar potential are identically zero for arbitrary expectation values. Each flat direction can be parameterized by some holomorphic invariant constructed out of the chiral superfields $I=Q_{1} Q_{2} \ldots Q_{N}$. Let $\varphi=$ $I^{1 / N}$ be a canonically normalized flat direction field. Generically, $Q_{i}$ are charged under the 


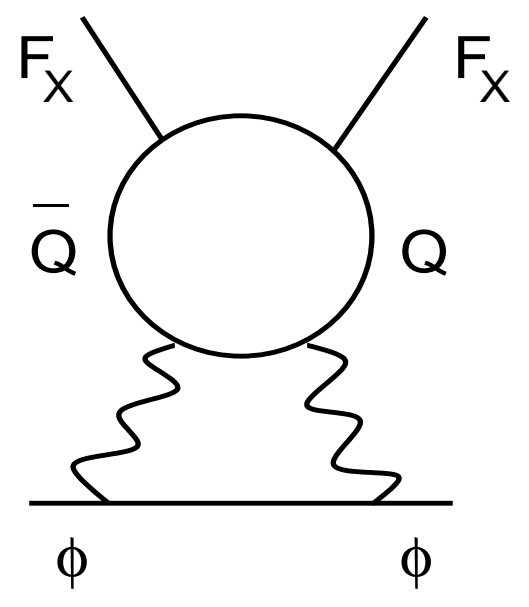

Figure 2: Supersymmetry breaking in the hidden sector can be communicated to $\varphi$ via messengers and gauge interactions.

standard model gauge group. Along the flat direction, all the gauge and chiral fields coupled to $\varphi$ get masses $\sim \varphi$ and become heavy. As the VEV becomes large, one can integrate them out and write an effective low energy theory. It will consist of the massless chiral superfield $\varphi$ which is essentially decoupled from the rest of the light particles. The rest of the interactions affect the massless field $\varphi$ only through some higher-dimensional operators suppressed by $\langle\varphi\rangle$. In addition, the supergravity corrections induce similar operators suppressed by $M_{P}$, which become important for $\langle\varphi\rangle \sim M_{P}$. Thus it is not surprising that in theories with low-energy supersymmetry breaking the potential becomes essentially flat for $\varphi \gg M$, up to the corrections due to gravity, which can lift it for very large field values.

For example, let us consider the case of gauge-mediated supersymmetry breaking. In this case the supersymmetry breaking is communicated to the observable sector, which includes $\varphi$ and other fields, by gauge interactions via a diagram shown in Figure 2. Here $Q$ and $\bar{Q}$ are the messengers, chiral superfields that share some common gauge interaction with $\varphi$. The corresponding gauge superfield is propagating in the lower loop. By assumption, there is also a tree-level coupling of messengers to the $X$ superfield in the superpotential, $X \bar{Q} Q$. They acquire a supersymmetric mass $\sim\langle X\rangle$ from this coupling. At the low energy scales, or, equivalently, for $\varphi<\langle X\rangle^{1 / 2}$, the flat potential for $\varphi$ is lifted by a soft mass term $\sim\left(\frac{\alpha}{4 \pi}\right)\left(\left\langle F_{X}\right\rangle /\langle X\rangle\right)$, the gauge fields in Figure 2 being light. However, farther out along the $\varphi$ direction, the VEV of $\varphi$ induces a mass of order $\langle\varphi\rangle$ for the gauge superfields 
in the two-loop diagram (Figure 2), which becomes dominated by the $1 /\langle\varphi\rangle^{2}$ factors from integrating over the gauge fields momenta in the lower loop. Therefore, at the scales (field strengths) much larger than $\langle X\rangle^{1 / 2}$, the soft mass of the $\varphi$ field gradually turns off, and the effective potential approaches zero asymptotically. Eventually, the Planck-scale corrections are expected to modify $U(\varphi)$ in the limit $\langle\varphi\rangle \rightarrow M_{P}$ and lift the the potential above zero. In general, there can be a variety of other minima in this region, which we will ignore. In any case, a qualitative behavior of the classically-flat potential in models with gauge-mediated (or any other low-energy) supersymmetry breaking is as shown in Figure 1.

\section{Toy model}

As a toy model, let's consider a theory with a scalar $\varphi$ and two light fermions, $q$ and $l$, with different masses $m_{q}>m_{l}$. The relevant part of the Lagrangian is

$$
V=U(\varphi)+y \frac{\varphi^{\dagger} \varphi}{M_{X}}(\bar{q} l)+h . c .,
$$

where the potential $U(\varphi)$, of the kind specified in the previous sections and shown in Figure 1, is augmented by an effective coupling to fermions suppressed by the large mass scale $M_{X} \gg$ $m_{\varphi}$ (such non-renormalizable interaction can arise naturally in realistic theories, as shown below). By assumption, $U(\varphi)$ is invariant under a global $\mathrm{U}_{\varphi}(1)$ symmetry $\varphi \rightarrow e^{i \theta} \varphi$. In addition, for $y=0$ there is a "baryon" $\mathrm{U}_{B}(1)$ symmetry $q \rightarrow e^{i \alpha} q$, and an analogous "lepton" number conservation for $l \rightarrow e^{i \beta} l$. In vacuum, $\langle\varphi\rangle=0$ and the transition of $q$ into $l$, our toy version of "proton decay", is disallowed at tree level. The first contribution to the $q \rightarrow \bar{l} l l$ decay arises at the two-loop level and the rate is small: $\Gamma \sim\left(y m_{\varphi} / M_{X}\right)^{6} m_{q}$.

Due to the $\mathrm{U}_{\varphi}(1)$ symmetry, the theory admits stable Q-balls. Inside a Q-ball, a non-zero $\mathrm{VEV}$ of $\varphi$ enhances the probability of the $q \rightarrow l$ transition' and the heavy fermion, q, has the decay width

$$
\Gamma \sim y^{2} \frac{\langle\varphi\rangle^{2}}{M_{X}^{2}} m_{q}
$$

that can be large for a sufficiently large VEV of $\varphi$. One can, therefore, use the Q-ball interior to catalyze the "proton decay" $q \rightarrow l$ and utilize the energy released in this process.

\footnotetext{
1 We note in passing that the momentum conservation in such decay is accommodated by the Q-ball recoil: $q \rightarrow l+$ sound waves (see discussion in Ref. [2]).
} 
In addition, because of the $q-l$ mixing induced by the coupling of $q$ and $l$ to $\varphi$, a $q$-particle scattering off a large Q-ball can be reflected as an $l$-particle with a probability

$$
P \sim y^{2} \frac{\langle\varphi\rangle^{4}}{m_{q}^{2} M_{x}^{2}},
$$

thus providing conditions for converting incoming "protons" into "leptons".

As a variation on this model, one can consider a Yukawa interaction

$$
V=U(\varphi)+y \varphi(\bar{q} l)+h . c .,
$$

where the Yukawa coupling $y$ is very small. Now the $\mathrm{U}_{\varphi}(1)$ symmetry is approximate: it is

broken by the small Yukawa coupling. The corresponding Q-ball is as stable as the symmetry is good and can slowly evaporate by emitting light fermions.

\section{The MSSM and its extensions}

The assumptions we made about the particle content of the theory and the form of the effective potential can be naturally realized in theories with softly broken supersymmetry. To create a Q-ball useful for probing the new physics, one needs a complex scalar field that transforms under a global U(1) symmetry. If a large VEV of such scalar can facilitate a proton decay, then the same Q-ball will also be useful for the purposes of generating power by releasing the energy locked in the nucleons by the baryon number conservation.

In the MSSM, the supersymmetric partners of the quarks and leptons can form Q-balls whose stability (neglecting the effect of fermions) owes to the conservation of the baryon and lepton numbers [6]. Under certain conditions, such objects can be created in the early Universe and can precipitate a decay of a false vacuum even if the tunneling probability is negligible [0]. Squarks and sleptons are, therefore, natural candidates for creating a Q-ball in a laboratory. However, in the presence of the light fermions that carry the same charge, these solitons can evaporate by emitting quarks and leptons from the surface as discussed in Ref. [8]. The rate of such evaporation is proportional to the surface area, rather than the volume of a Q-ball, and can only be neglected if a sufficiently large Q-ball can be built. While not fatal in principle, this can be seen as a considerable complication.

To illustrate the general idea, we turn to the case in which the issue of stability is simplified by the absence of light U(1)-charged particles in the theory, and where the "flat" 
potential is realized in terms of colorless degrees of freedom. The considerations of stability are trivial if an additional scalar field, whose couplings to matter fermions are small, is used to create a soliton. Such fields, in fact, do appear in various extensions of the Standard Model.

An example of the light decoupled scalars can be provided by the color-triplet Higgs $(T, \bar{T})$ partners of the electroweak Higgs doublets. These particles are inevitably present in any GUT extension of the MSSM and carry a non-zero baryon and lepton number, since they are coupled to the ordinary quarks and leptons. To explain the proton stability, one usually assumes these particles to be very heavy. This is the well known doublet-triplet splitting problem in SUSY GUTs. The enormous mass splitting, however, can be avoided if the triplets are light but have very weak, GUT scale suppressed interactions with quarks and leptons [9]. Such suppression is due to Clebsch factors which are determined by the group structure of the the GUT-breaking VEVs. This can be achieved naturally, as in the model of Ref. 9]. Since the Higgs triplets are essentially decoupled (from quarks and leptons), the low energy action below the GUT scale contains an additional unbroken global symmetry $\mathrm{G}=U_{T}^{\prime}(1) \times U_{T}^{\prime \prime}(1)$ which transforms $T \rightarrow e^{i \theta} T$ and $\bar{T} \rightarrow e^{i \chi} \bar{T}$. This global symmetry is only broken by small couplings, suppressed by the powers of the GUT scale. In a theory with gauge-mediated supersymmetry breaking the triplets have soft masses of order $\sim 1 \mathrm{TeV}$ which vanish at energy scales large in comparison to the messenger mass. The corresponding potential has a flat direction for some combination $\varphi$ of $T$ and $\bar{T}$, charged under a subgroup $\mathrm{U}_{T}(1)$ of $\mathrm{G}$. This potential satisfies the conditions discussed in the previous section and is suitable for building $\mathrm{U}_{T}(1)$ Q-balls.

Depending on the cosmological history of the Universe, the triplets may or may not be subject to constraints related to the non-observation of the "wild hydrogen", and other exotic elements whose nuclei might contain a heavy stable colored $T$-particle. If inflation took place and ended with a relatively low reheating temperature, sufficiently below the $T$ mass, then no constraints on the mass and lifetime of the triplets arise. Otherwise, $T$ particles must decay in less than $10^{10}$ years to evade the superheavy element searches and other bounds [10]. Different considerations [11] exclude the (electrically charged) stable $T$ particles outside the 20 to $1000 \mathrm{TeV}$ window. In any case, even without the help of a relatively cool inflation, weakly-interacting $T$ particles with lifetimes $>10 \mathrm{~s}$ and masses in the $\mathrm{TeV}$ region are allowed 
by the data.

If the color triplets are discovered, they can be pair-produced in a hadron collider. If stable, the $T$ particles can also be found on Earth, although their concentration is constrained to be small [10]. We assume that the $T$-particles can be condensed into Q-balls which will grow as one feeds in some additional charge. Technical and engineering aspects of such process, which may or may not be possible to realize in practice, lie outside the scope of our investigation.

There are two obvious candidates for building stable Q-balls in this model. We assume that the soft masses for both the squark $q$ and the $T$-field originate at the scale $M_{S}$ and effectively turn off at higher energy scales, as explained in section 2. The effective potential in terms of each of the colorless fields $\varphi_{1}=\left(T^{a} \tilde{q}^{a}\right)^{1 / 2}$ and $\varphi_{2}=\left(T^{a} \bar{T}^{a}\right)^{1 / 2}$ is then of the form specified above, with a nearly flat direction. Both $\varphi_{1}$ and $\varphi_{2}$ are suitable for Q-balls. Below we will often use $\varphi$ for either of the two fields. The field $\varphi$ inside the Q-ball develops a large VEV limited only by the Q-ball size. The increase of a scalar VEV inside a growing Q-ball can eventually trigger some exotic processes characteristic of the new physics at the high energy scale. Presumably, it would take only a TeV-scale machine to produce the $T$ particles. However, if a large enough Q-ball is created, one can explore the new frontiers well above the TeV energy scale.

If the scalar field that makes up a Q-ball can decay into quarks, then there is a finite density of quarks (of order $m_{\varphi}^{3}$ ) inside the Q-ball. The interior of such Q-ball is similar in nature to the cold but dense quark-gluon plasma. This is not the case if $\varphi$ particles are stable. If the scalar VEV is sufficiently large, the electroweak symmetry may be restored inside the Q-ball. For the $\mathrm{SU}(2)$-nonsinglet fields $\varphi$ the running of couplings is frozen at the scale $\mu \sim \varphi$, which may correspond to an unbroken $\mathrm{SU}(2) \times \mathrm{U}(1)$.

For example, in theories with gauge-mediated supersymmetry breaking, the soft mass of the Higgs boson originates below the scale $M_{S}$ at the two-loop level:

$$
m_{H}^{2}=\mu^{2}+\left[c_{1}\left(\frac{\alpha_{1}}{4 \pi}\right)^{2}+c_{2}\left(\frac{\alpha_{2}}{4 \pi}\right)^{2}\right] \frac{F_{X}^{2}}{M_{S}^{2}}
$$

where $\alpha_{i}$ are the gauge couplings and $c_{1}$ and $c_{2}$ are the group factors. The negative contribution to the Higgs mass, necessary for the electroweak symmetry breaking, comes at the three-loop level due to the strong Yukawa interaction with the top: 


$$
\Delta m_{H}^{2}=-\frac{6 h_{t}^{2}}{16 \pi^{2}} \ln \left(\frac{\Lambda}{\tilde{m}_{t}}\right) \tilde{m}_{t}^{2}
$$

where $h_{t}$ is the top Yukawa coupling, and $\tilde{m}_{t}^{2} \sim\left(\frac{\alpha_{3}}{4 \pi}\right)^{2} \frac{F_{X}^{2}}{M_{S}^{2}}$ is the two-loop mass of the stop. This contribution can be dominant, thanks to the large value of $\alpha_{3}$.

If the VEV's of $T$ and $\tilde{q}$ are large inside the Q-ball built of $T$-fields and the righthanded ( $\mathrm{SU}(2)$-singlet) squarks, then $\alpha_{3}$ is small. The negative contribution in equation (11) diminishes and $m_{H}^{2}$ can become positive in the Q-ball interior. Thus, the electroweak symmetry is restored and, in particular, the sphaleron transitions can go unsuppressed. In this sense the ground state inside the Q-ball is similar to that of the electroweak bags [12].

We now want to examine the feasibility of using Q-balls for power generation via the nucleon decay. There are several processes that can yield large amounts of energy once a Q-ball is assembled and placed in a beam of protons.

First, the dissociation of protons into quarks can yield $\sim 1 \mathrm{GeV}$ of energy per nucleon. For a large VEV of $\varphi$ the QCD coupling becomes small. As a result, an incoming proton will dissociate into quarks and dissipate the binding energy $\sim 1 \mathrm{GeV}$ in photons and "sound waves" [2]. These excitations can propagate to the Q-ball surface, where the energy is emitted in photons and pions pair-produced near the soliton boundary.

Second effect is the sphaleron transitions of quarks into leptons, which are possible if the $\mathrm{SU}(2)_{L} \times \mathrm{U}(1)$ symmetry is restored inside the Q-ball, much like in the case of the electroweak bags 12 .

Third, as in the toy model of section 3, the incoming protons can scatter off the Q-ball boundary as leptons. The T VEV can induce a mixing between quarks and leptons [9]. If $\langle T\rangle$ is large, the mass eigenvalues for quarks and leptons inside the Q-ball can be larger than those in vacuum. A soliton boundary can then serve as a repulsive potential barrier for protons. Due to the large quark-lepton mixing in the barrier, the back-scattered fermions will be leptons with non-vanishing probability.

Proton decay catalyzed by the presence of the Grand Unified magnetic monopoles [13] has been considered as a potential source of energy. It was estimated [14] that $10^{11}$ monopoles would be needed to replace one industrial power plant. In the case of Q-balls, the efficiency of power generation would depend on their size. For instance, a single Q-ball placed in water 
would catalyze proton decay at the rate proportional to its surface area. An "industrial" amount of power $\sim 10^{8} \mathrm{~W}$ could be achieved with the charge $10^{40}$ Q-ball, a few microns in diameter, weighing 100 tons. The same efficiency can be achieved using $10^{17}$ Q-balls with charge $10^{6}$ each, hence reducing the total charge to $10^{23}$. Although the surface-to-volume ratio favors a higher number of smaller Q-balls, there is a model-dependent limit on the charge of the smallest soliton that catalyzes a proton decay in one of the way discussed above.

\section{Conclusion}

Scalar particles, such as squarks, sleptons, and others, may be discovered at the scales $\lesssim 1 \mathrm{TeV}$, which will become accessible to particle accelerators in the near future. The new scalar fields may transform non-trivially under $B, L$, or some new global symmetries and may have the requisite couplings [6] suitable for Q-balls.

If, as expected in SUSY theories, the scalar potential has flat directions, then the Qball interior may provide the environment for exploring physics far beyond the $\mathrm{TeV}$ scale without the need for building colliders of such enormous energies. Instead, one can learn to create large Q-balls in a laboratory setting, and use them as instruments for high-energy experimentation. The exotic processes that can take place inside a large artificially-built Q-ball can be detected and analyzed, thus helping elucidate the new physics.

Normally suppressed, baryon number violating processes may become unleashed inside the Q-ball. A controlled proton decay catalyzed by a Q-ball can serve as an inexhaustible source of energy. Although speculative, this idea requires few assumptions about the physics beyond the Standard Model: we have assumed the MSSM with low-energy supersymmetry breaking. As was explained earlier, some additional scalars with new conserved U(1) quantum numbers can be of help, but are not necessary.

We thank A. Cohen, S. Coleman and P. Tinyakov for helpful discussions. 


\section{References}

[1] T. D. Lee and Y. Pang, Phys. Rep. 221 (1992) 251, and references therein.

[2] S. Coleman, Nucl. Phys. B262 (1985) 263.

[3] For review, see, e.g., S. Dimopoulos, M. Dine, S. Raby, S. Thomas, J. D. Wells, Nucl. Phys. Proc. Suppl. A52 (1997) 38, and references therein.

[4] A. Kusenko, Phys. Lett. B404 (1997) 285.

[5] A. D. Linde, Nucl. Phys. B216 (1983) 421.

[6] A. Kusenko, Phys. Lett. B405 (1997) 108.

[7] A. Kusenko, Phys. Lett. B, in press (hep-ph/9705361).

[8] A. Cohen, S. Coleman, H. Georgi and A. Manohar, Nucl. Phys. B272 (1986) 301.

[9] G. R. Dvali, Phys. Lett. B287 (1992) 101; ibid. B372 (1996) 113.

[10] V. A. Kuzmin and M. E. Shaposhnikov, Nuovo Cim. 82A (1984) 35; E. Nardi and E. Roulet, Phys. Lett. B245 (1990) 105.

[11] A. De Rújula, S. L. Glashow and U. Sarid, Nucl. Phys. B333 (1990) 173.

[12] S. Yu. Khlebnikov and M. E. Shaposhnikov, Phys. Lett. B180 (1986) 93, Sov. J. Nucl. Phys. 45 (1987) 747.

[13] V. A. Rubakov, JETP Lett. 33 (1981) 644, Nucl. Phys. B203 (1982) 311; C. G. Callan, Phys. Rev. D25 (1982) 2141.

[14] F. A. Bais, J. Ellis, D. V. Nanopoulos and K. A. Olive, Phys. Lett. B116 (1982) 127; Nucl. Phys. B219 (1983) 189. 